Communications in Physics, Vol. 24, No. 3 (2014), pp. 217-224

DOI:10.15625/0868-3166/24/3/3915

\title{
EIT ENHANCED SELF-KERR NONLINEARITY IN THE THREE-LEVEL LAMBDA SYSTEM UNDER DOPPLER BROADENING
}

\author{
DINH XUAN KHOA, LE VAN DOAI, PHAM VAN TRONG, TRAN MANH CUONG, \\ VU NGOC SAU, AND NGUYEN HUY BANG \\ Vinh University, 182 Le Duan Street, Vinh City, Nghe An, Vietnam \\ LE NGUYEN MAI ANH \\ Nong Lam University - Thu Duc district - Ho Chi Minh City - Vietnam \\ E-mail: bangvinhuni@gmail.com \\ Received 17 April 2014 \\ Accepted for publication 30 August 2014
}

\begin{abstract}
Using density-matrix theory, an analytical expression of the self-Kerr nonlinear coefficient of a three-level lambda EIT medium for a weak probe light is derived. Influences of the coupling light and Doppler broadening on the self-Kerr coefficient are investigated and compared to experimental observation with a good agreement. The selfKerr nonlinearity of the medium is modified and greatly enhanced in the spectral region of EIT window. Furthermore, sign, slope, and magnitude of the self-Kerr coefficient can be controlled with frequency and intensity of the coupling light and temperature of the medium. Specially, for a given set of fixed values of the parameters of coupling and probe lights, it could be able to choose an optimized temperature to have largest magnitude of the self-Kerr coefficient. Such controllable Kerr nonlinearity can find interesting applications in optoelectronic devices working with low-light intensity at various temperature conditions.
\end{abstract}

Keywords: Kerr nonlinearity; atomic coherence; electromagnetically induced transparency.

\section{INTRODUCTION}

The Kerr nonlinear materials are used widely in photonic devices and multi-wave mixing converters. In most cases, a strong nonlinear response is often needed to gain conversion efficiency. However, due to weakness of Kerr nonlinearity of traditional optical materials, several theoretical proposals have not yet been experimentally observed. Finding materials having large Kerr nonlinearity is therefore of interest to realize nonlinear processes at low-light intensities. A proposal to achieve this goal is to use lights having frequencies matched with atomic resonances under electromagnetically induced transparency (EIT) $[1,2]$. As have been shown in literatures, in addition to the reduction of absorption, the behavior of steeper dispersion that leads to greatly lengthen interaction time, EIT media therefore become ideal for the applications needed materials having large nonlinear response [2,3].

In the recent years, we have seen a tremendous progress of study related to EIT enhanced Kerr nonlinearity due to it concerns several interesting and potential applications. Some typical (C)2014 Vietnam Academy of Science and Technology 
applications among of these are cross-phase modulation for optical shutters [4], self-phase modulation for generating optical solitons [5], four-wave mixing processes for frequency conversion [6], and entangled states for quantum information processing [7]. The basic configuration for EIT enhanced Kerr nonlinearity is three-level systems that induces large nonlinear response in a narrow spectral region $[2,8,9]$.

Of particular interest to the present work, Wang et al., [8] performed, for the first time, a direct measurement of self-Kerr coefficient in a three-level lambda EIT medium in the present of Doppler broadening by using the ring cavity scanning technique. The measurement showed a great enhancement of the Kerr nonlinear coefficient, and explained with numerical simulations by the same authors. Although the numerical simulations were helpful to explain experimental observation but there still lack of analytical representation of the self-Kerr nonlinear coefficient. Such shortage of analytical result has hampered implementation of further studies of nonlinear processes, for example, optimizing temperature at a certain set of values of parameters of the coupling and probe lights to have largest nonlinear efficiency. As in several applications related to such Kerr medium [9], a precise knowledge of the third-order susceptibility or the Kerr nonlinear coefficient $n_{2}$ as a function of coupling light and temperature is needed to optimize nonlinear optical processes. Up to date, to our best knowledge, nevertheless, the Kerr nonlinear coefficient of the three-level EIT medium in the present of Doppler broadening has not yet been derived in any analytical form.

In this work, using dipole and rotating approximations, we derive the third-order nonlinear susceptibility and the self-Kerr coefficient of the three-level lambda EIT medium in the present of Doppler broadening by using an iterative perturbation method. The influences of Doppler broadening and coupling light on magnitude, sign, and slope of the Kerr coefficient are investigated and compared to the recent experimental observation.

\section{THEORETICAL MODEL}

We consider a three-level lambda system as shown in Fig. 1, as in Ref. [8]. A weak probe laser beam, with frequency $\omega_{p}$, drives the transition $|1\rangle \rightarrow|2\rangle$ with Rabi frequency $\Omega_{p}$, whereas an intense coupling laser beam (with frequency $\omega_{c}$ and Rabi frequency $\Omega_{c}$ ) couples a transition between the state $|2\rangle$ and $|3\rangle$. The frequency detuning of the coupling and probe laser is respectively defined as:

$$
\Delta_{p}=\omega_{p}-\omega_{21}, \Delta_{c}=\omega_{c}-\omega_{23} .
$$

In the framework of the semiclassical theory, using the dipole and rotating wave approximations, evolution of the system can be represented by the following density-matrix equations [8]

$$
\begin{gathered}
\dot{\rho}_{11}=\gamma_{31}\left(\rho_{33}^{-} \rho_{11}^{)}+\gamma_{21} \rho_{22}-\frac{i}{2} \Omega_{p} \rho_{21}+\frac{i}{2} \Omega_{p} \rho_{12},\right. \\
\dot{\rho}_{22}=-\left(\gamma_{23}+\gamma_{21}\right) \rho_{22}-\frac{i}{2} \Omega_{p} \rho_{12}+\frac{i}{2} \Omega_{p} \rho_{21}-\frac{i}{2} \Omega_{c} \rho_{32}+\frac{i}{2} \Omega_{c} \rho_{23}, \\
\dot{\rho}_{33}=\gamma_{31}\left(\rho_{11}-\rho_{33}\right)+\gamma_{23} \rho_{22}+\frac{i}{2} \Omega_{c} \rho_{32}-\frac{i}{2} \Omega_{c} \rho_{23}, \\
\dot{\rho}_{21}=-\left(\gamma-i \Delta_{p}\right) \rho_{21}^{+} \frac{i}{2} \Omega_{p}\left(\rho_{22}-\rho_{11}\right)-\frac{i}{2} \Omega_{c} \rho_{31},
\end{gathered}
$$




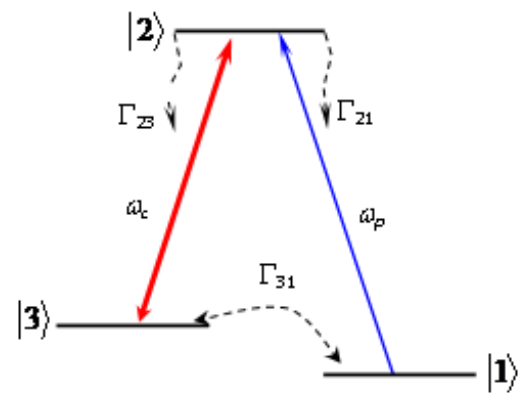

Fig. 1. The three-level $\Lambda$-type system.

$$
\begin{gathered}
\dot{\rho}_{23}=-\left(\gamma-i \Delta_{c}\right) \rho_{23}+\frac{i}{2} \Omega_{c}\left(\rho_{22}-\rho_{33}\right)-\frac{i}{2} \Omega_{p} \rho_{13}, \\
\dot{\rho}_{31}=-\left[\gamma_{31}-i\left(\Delta_{p}-\Delta_{c}\right)\right] \rho_{31}+\frac{i}{2} \Omega_{p} \rho_{32}-\frac{i}{2} \Omega_{c} \rho_{21},
\end{gathered}
$$

where $\gamma_{i k}$ is represented with the decay rates $\Gamma_{i k}$ from $|i\rangle$ to $|k\rangle$ as follows

$$
\gamma_{i j}=\frac{1}{2}\left(\sum_{k<i} \Gamma_{i k}+\sum_{l<j} \Gamma_{j l}\right),
$$

and

$$
\gamma=\frac{\gamma_{21}+\gamma_{31}+\gamma_{23}}{2}
$$

In the Eqs.(2) - (7), the diagonal matrix elements $\rho_{i i}(i=1 \div 3)$ represent population in the state $|i\rangle$, whereas the off-diagonal matrix elements $\rho_{i j}(i \neq j)$ represent the coherence between the states $|i\rangle$ and $|j\rangle$; the matrix-elements are hermitic, namely, $\rho_{i j}=\rho_{j i}^{*}$.

Using perturbation theory, density-matrix elements are represented by [8]

$$
\rho_{\mathrm{ij}}=\rho_{\mathrm{ij}}^{(0)}+\rho_{\mathrm{ij}}^{(1)}+\cdots \rho_{\mathrm{ij}}^{(n)},
$$

where, each successive approximation is calculated using the density-matrix elements of one order less than the one being calculated. On the other hand, due to the conservation of probability, we assume

$$
\rho_{11}^{(0)}+\rho_{22}^{(0)}+\rho_{33}^{(0)}=1 .
$$

In the weak-field limit of the probe light, most of the initial population is assumed in the ground state $|1\rangle$, namely:

$$
\rho_{11}^{(0)} \approx 1, \quad \rho_{22}^{(0)} \approx 0, \quad \rho_{33}^{(0)} \approx 0 .
$$

Following above assumptions, we obtain $\rho_{21}$ from Eqs. (5) and (7), to the first-order as

$$
\rho_{21}^{(1)}=\frac{\frac{i}{2} \Omega_{p}\left(\rho_{22}^{(0)}-\rho_{11}^{(0)}\right)}{F} \approx \frac{-\frac{i}{2} \Omega_{p}}{F},
$$

where

$$
F=\gamma-i \Delta_{p}+\frac{\left(\Omega_{c} / 2\right)^{2}}{\gamma_{31}-i\left(\Delta_{p}-\Delta_{c}\right)}
$$


In a similar way, the third-order of $\rho_{21}$ is derived as

$$
\rho_{21}^{(3)}=\frac{\frac{i}{2} \Omega_{p}\left(\rho_{22}^{(2)}-\rho_{11}^{(2)}\right)}{F} .
$$

With assuming $\rho_{33}^{(2)} \approx 0$, we have

$$
\rho_{22}^{(2)}-\rho_{11}^{(2)}=\frac{2}{2 \gamma+\gamma_{21}}\left[i \Omega_{p} \rho_{21}^{(1)}-i \Omega_{p} \rho_{12}^{(1)}\right]+\frac{2 \gamma_{31}}{2 \gamma+\gamma_{21}} .
$$

By inserting Eq.(16) into Eq.(15) we obtain the third-order matrix element $\rho_{21}^{(3)}$ as:

$$
\rho_{21}^{(3)}=\frac{i \Omega_{p}}{2 F}\left(\frac{\Omega_{p}^{2}}{2 \gamma+\gamma_{21}}\left[\frac{1}{F}+\frac{1}{F^{*}}\right]+\frac{2 \gamma_{31}}{2 \gamma+\gamma_{21}}\right) .
$$

Having $\rho_{21}^{(1)}$ and $\rho_{21}^{(3)}$, the matrix element $\rho_{21}$ is determined to third-order as

$$
\rho_{21}=\rho_{21}^{(1)}+\rho_{21}^{(3)}=\frac{-i \Omega_{p}}{2 F}\left(1-\frac{2 \gamma_{31}}{2 \gamma+\gamma_{21}}\right)+\frac{i \Omega_{p}}{2 F} \frac{\Omega_{p}^{2}}{2 \gamma+\gamma_{21}}\left(\frac{1}{F}+\frac{1}{F^{*}}\right),
$$

where, $F^{*}$ is the complex conjugation of $F$.

The total susceptibility $\chi$ for the probe light is represented by

$$
\chi=-2 \frac{N d_{21}}{\varepsilon_{0} E_{p}} \rho_{21}=\frac{N d_{21}}{\varepsilon_{0} E_{p}}\left[\frac{i \Omega_{p}}{F}\left(1-\frac{2 \gamma_{31}}{2 \gamma+\gamma_{21}}\right)-\frac{i \Omega_{p}}{F} \frac{\Omega_{p}^{2}}{2 \gamma+\gamma_{21}}\left(\frac{1}{F}+\frac{1}{F^{*}}\right)\right] .
$$

In order to extract the third-order nonlinear susceptibility we interpret the total susceptibility in Eq.(19) in an alternative form

$$
\chi=\chi^{(1)}+3 E_{p}^{2} \chi^{(3)} .
$$

From Eqs.(19) and (20) we can derived the linear susceptibility $\chi^{(1)}$ and the third-order nonlinear susceptibility $\chi^{(3)}$ as

$$
\begin{gathered}
\chi^{(1)}=\frac{i N d_{21}^{2}}{\varepsilon_{0} \hbar} \frac{1}{F}\left(1-\frac{2 \gamma_{31}}{2 \gamma+\gamma_{21}}\right), \\
\chi^{(3)}=-\frac{i N d_{21}^{4}}{3 \varepsilon_{0} \hbar^{3}} \frac{1}{2 \gamma+\gamma_{21}} \frac{1}{F}\left(\frac{1}{F}+\frac{1}{F^{*}}\right) .
\end{gathered}
$$

For a vapor medium, it is necessary taken into account Doppler broadening. As in several experimental conditions for this type of configuration, we consider the co-propagation of the probe and coupling beams in the medium. Therefore, a particle moving with velocity $v$ towards the propagation direction of the probe beam will see an up-shift frequencies of the probe and coupling laser as $\omega_{p}+(v / c) \omega_{p}$ and $\omega_{c}+(v / c) \omega_{c}$, respectively. In such case, the linear and third-order nonlinear susceptibilities must be modified to

$$
\begin{gathered}
\chi^{(1)}(v) d v=\frac{i N_{0} d_{21}^{2}}{u \sqrt{\pi} \varepsilon_{0} \hbar}\left(1-\frac{2 \gamma_{31}}{2 \gamma+\gamma_{21}}\right) \frac{e^{-v^{2} / u^{2}}}{F(v)} d v \\
\chi^{(3)}(v) d v=-\frac{i N_{0} d_{21}^{4}}{3 u \sqrt{\pi} \varepsilon_{0} \hbar^{3}}\left(\frac{1}{2 \gamma+\gamma_{21}}\right) \frac{e^{-v^{2} / u^{2}}}{F(v)}\left[\frac{1}{F(v)}+\frac{1}{F^{*}(v)}\right] d v
\end{gathered}
$$


where, $u=\sqrt{2 k_{B} T / m}$, and

$$
F(v)=\gamma-i\left(\Delta_{p}+\frac{v}{c} \omega_{p}\right)+\frac{\Omega_{c}^{2} / 4}{\gamma_{31}-i\left(\Delta_{p}-\Delta_{c}\right)-i \frac{v}{c}\left(\omega_{p}-\omega_{c}\right)} .
$$

Since $\omega_{p}$ is close to $\omega_{c}$ so term $i \frac{v}{c}\left(\omega_{p}-\omega_{c}\right)$ in Eq.(25) can be neglected. By integrating Eqs. (23) and (24) over velocity distribution, we obtain:

$$
\begin{gathered}
\chi^{(1)}=\frac{i N_{0} d_{21}^{2} \sqrt{\pi}}{\varepsilon_{0} \hbar\left(\omega_{p} u / c\right)}\left(1-\frac{2 \gamma_{31}}{2 \gamma+\gamma_{21}}\right) e^{z^{2}}[1-\operatorname{erf}(z)], \\
\chi^{(3)}=-\frac{i N_{0} d_{21}^{4}}{3 \sqrt{\pi} \varepsilon_{0} \hbar^{3}\left(\omega_{p} u / c\right)^{2}}\left(\frac{1}{2 \gamma+\gamma_{21}}\right) \\
\times\left\{2 \sqrt{\pi}\left(-1+\sqrt{\pi} z e^{z^{2}}[1-\operatorname{erf}(z)]\right)+\frac{\pi\left(e^{z^{2}}[1-\operatorname{erf}(z)]+e^{z^{* 2}}\left[1-\operatorname{erf}\left(z^{*}\right)\right]\right)}{z+z^{*}}\right\},
\end{gathered}
$$

where

$$
z=\frac{c}{\omega_{p} u}\left(\gamma-i \Delta_{p}+\frac{\Omega_{c}^{2} / 4}{\gamma_{31}-i\left(\Delta_{p}-\Delta_{c}\right)}\right)=\frac{c}{\omega_{p} u} F,
$$

and $z^{*}$ represents the complex conjugation of $z$, and $\operatorname{erf}$ is error function.

Having the linear and third-order nonlinear susceptibilities, the nonlinear Kerr index of refraction $n_{2}$ is determined according to the following relations [9]:

$$
n_{2}=\frac{3 \operatorname{Re}\left(\chi^{(3)}\right)}{4 \varepsilon_{0} n_{0}^{2} c}, \quad n_{0}=\sqrt{1+\operatorname{Re}\left(\chi^{(1)}\right)} .
$$

The first expression in (29) represents the self-Kerr nonlinear coefficient for the probe light as a function of controllable parameters, intensity and frequency detuning of the coupling light.

\section{ANALYSIS OF KERR NONLINEARITY}

The expression of $n_{2}$ obtained above provides an overall view to see continuous variation of the self-Kerr nonlinear coefficient of the three-level lambda system with respect to controllable parameters of the coupling light and Doppler broadening. In principle, the expression can generally be applied to atomic and/or molecular systems having spectroscopic structure similar to that presented in Fig. 1. As a simple illustration, we visualize the analytical result to the medium of ${ }^{87} \mathrm{Rb}$ atomic vapor. In this case, the states $|1\rangle,|2\rangle$, and $|3\rangle$ are chosen as $5 S_{1 / 2}(F=1), 5 P_{1 / 2}(F=2)$, and $5 S_{1 / 2}(F=2)$, respectively. We choose the atomic parameters by [8]: $N=4.5 \times 10^{17}$ atoms $/ \mathrm{m}^{3}$; $\gamma_{21}=2 \pi \times 3 \mathrm{MHz}, \gamma_{31}=2 \pi \times 1.1 \mathrm{MHz}$, and $\gamma=2 \pi \times 3.5 \mathrm{MHz} d_{21}=1.6 \times 10^{-29} \mathrm{C} . \mathrm{m} \mathrm{[10]}$.

The first step, we consider influence of EIT on Kerr nonlinearity by comparing the value of $n_{2}$ between the present and absent of EIT. The later is simply obtained by setting Rabi frequency $\Omega_{c}=0$ in Eq. (29). Fig. 2(a) shows variation of the Kerr nonlinear coefficient $n_{2}$ in both cases versus the probe frequency detuning. It is apparently to see a fundamental modification and a great enhancement of the self-Kerr nonlinear coefficient in the three-level system under the present of the EIT effect. Such behavior of the obtained analytical result is tested by comparing with experimental observation in Ref. [8] as shown in Fig. 2(b). The comparison confirms a good quality of the theoretical derivation. 

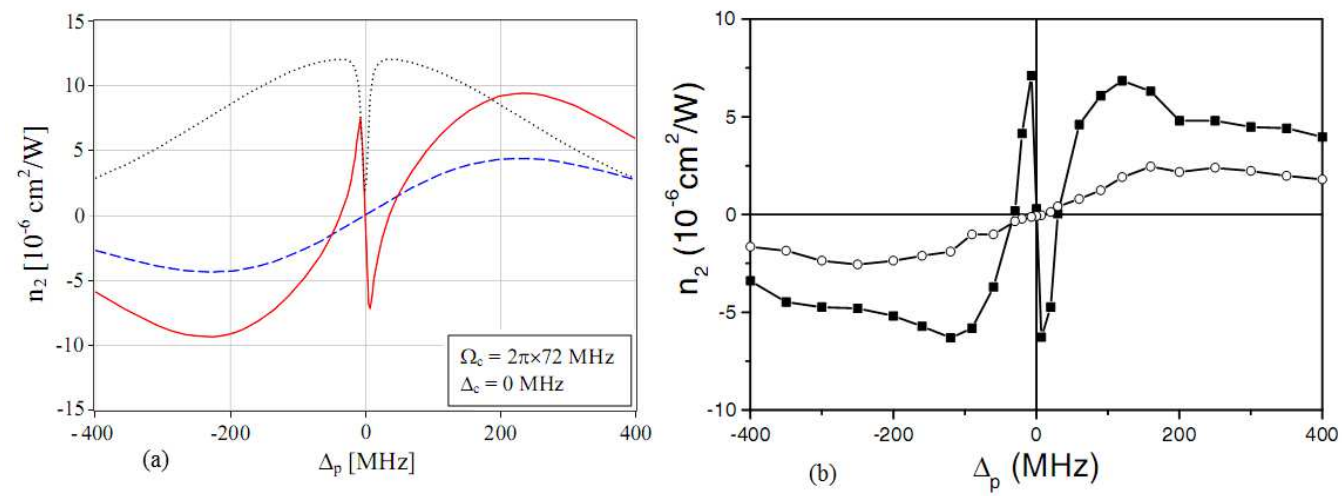

Fig. 2. (a) The variation of Kerr nonlinear coefficient $n_{2}$ versus probe detuning $\Delta_{p}$ for $\Delta_{c}=0$ in the present (dashed curve) and absent (solid curve) of the EIT effect at temperature $336 \mathrm{~K}$. The dotted line represents EIT spectrum of the three-level system determined by the imaginary part of Eq. (26). (b) Experimental measurement of $n_{2}$ in the Ref. [8] for $\Delta_{c}=0$ and $\Omega_{c}=2 \pi \times 72 \mathrm{MHz}$, where the square- and circular- curves stand for the present and absent of the coupling light, respectively.

In order to study influence the coupling light intensity on the Kerr coefficient, we consider the case where the coupling light is on resonance, and probe frequency is fixed at $\Delta_{p}= \pm 7 \mathrm{MHz}$, which correspond the maximum and minimum points (locate in the spectral region of the EIT window, represent by the dotted line in Fig.2a) on the profile of $n_{2}$ (solid line in Fig.2a). The variation of the Kerr nonlinear coefficient versus the Rabi frequency of the coupling light is shown in Fig. 3. Such variation shows that one may control magnitude of the Kerr nonlinear coefficient by tuning intensity of the coupling light.
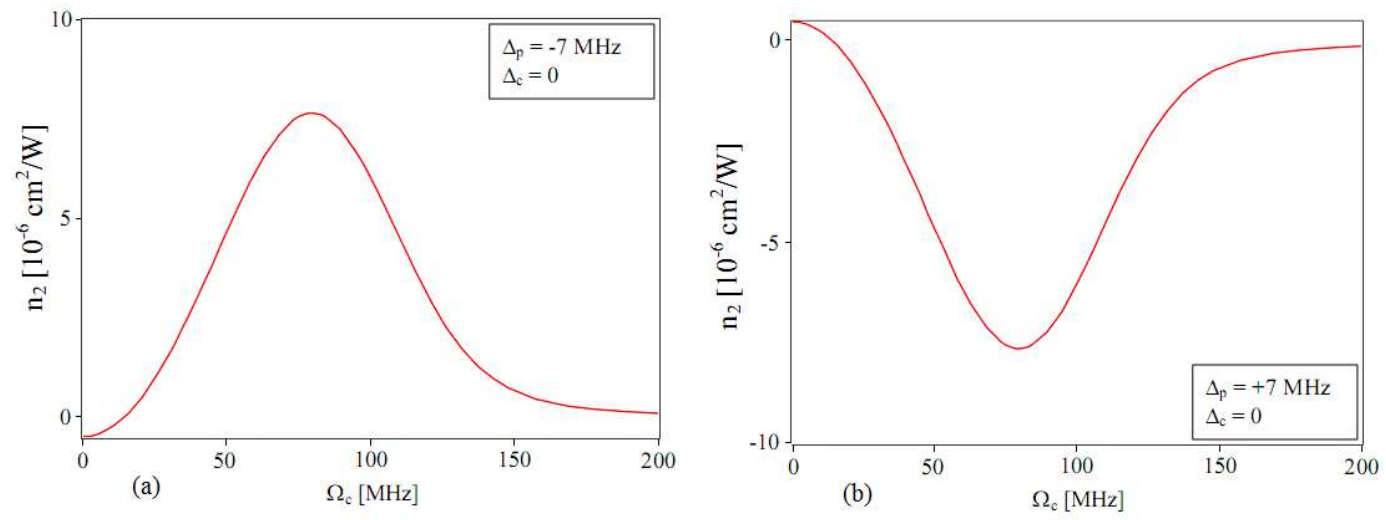

Fig. 3. Variation of the Kerr nonlinear coefficient $n_{2}$ versus the coupling intensity $\Omega_{c}$ when $\Delta_{c}=0$ for the cases of $\Delta_{p}=-7 \mathrm{MHz}$ (a) and $\Delta_{p}=+7 \mathrm{MHz}$ (b). 
In addition to the influence of the intensity, the influence of frequency of the coupling light on the Kerr nonlinear coefficient is considered by investigation the variation of $n_{2}$ with respect to frequency detuning $\Delta_{c}$. We choose a zero value of $n_{2}$ corresponding to $\Delta_{p}=0$ and $\Omega_{c}=2 \pi \times 72$ $\mathrm{MHz}$ as in Fig.2(a). Variation of the Kerr coefficient with respect to frequency detuning of the coupling light is shown in Fig.4a. The behavior shows that the Kerr coefficient can be changed not only in magnitude but also in its sign.
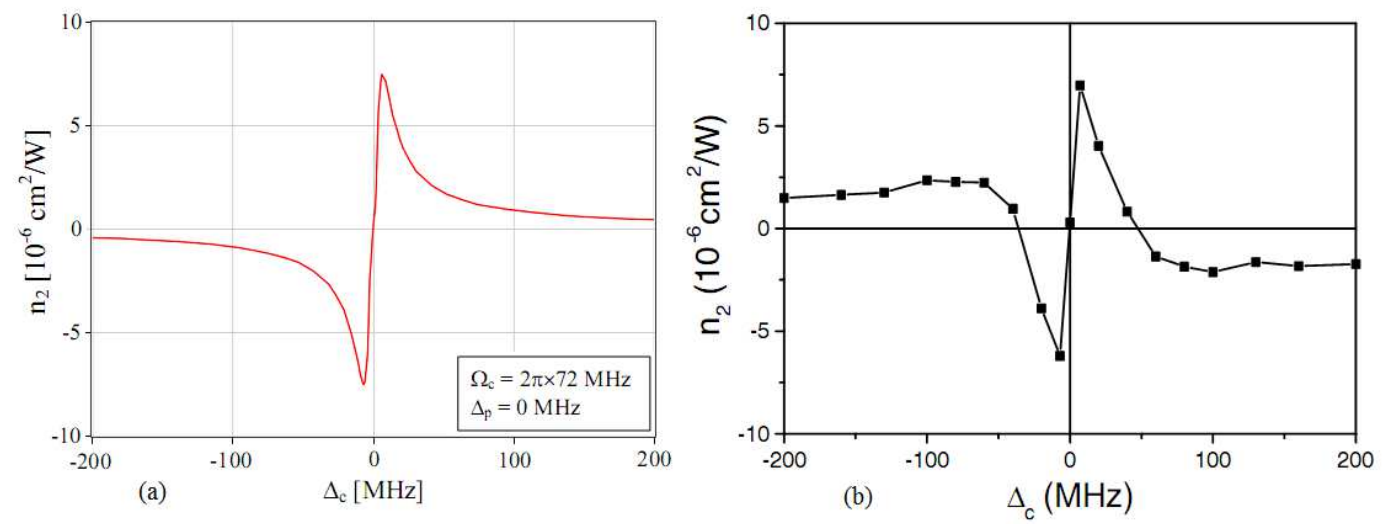

Fig. 4. Change of sign and magnitude of the Kerr nonlinear coefficient $n_{2}$ with respect to the frequency detuning of the coupling light for the case where $\Delta_{p}=0$ and $\Omega_{c}=2 \pi \times 72$ MHz. Figures (a) and (b) represent theoretical calculation in this work and experimental measurement in Ref. [8], respectively.

Finally, we study influence of temperature on magnitude of the Kerr coefficient. We choose the maximum point of $n_{2}$ corresponding to parameters $\Delta_{c}=0, \Delta_{p}=-7$ $\mathrm{MHz}$, and $\Omega_{c}=2 \pi \times 72 \mathrm{MHz}$, as indicated by the solid line in Fig. 2a. Variation of $n_{2}$ with respect to absolute temperature at the fixed parameters is shown in Fig. 5.

The Fig. 5 shows that, for a given set of fixed values of the parameters $\Delta_{c}, \Delta_{p}$, and $\Omega_{c}$ it could be able to choose an optimized temperature to have largest value of the self-Kerr coefficient $n_{2}$. For the set of values indicated in Fig. 5, the absolute temperature for largest value of $n_{2}$ is approximately $57 \mathrm{~K}$. The maximum value of $n_{2}$ in this case is $28 \times 10^{-6} \mathrm{~cm}^{2} / \mathrm{W}$ that is much larger than the case in Fig. $2 \mathrm{a}$ where $n_{2}=$ $8 \times 10^{-6} \mathrm{~cm}^{2} / \mathrm{W}$ at $336 \mathrm{~K}$.

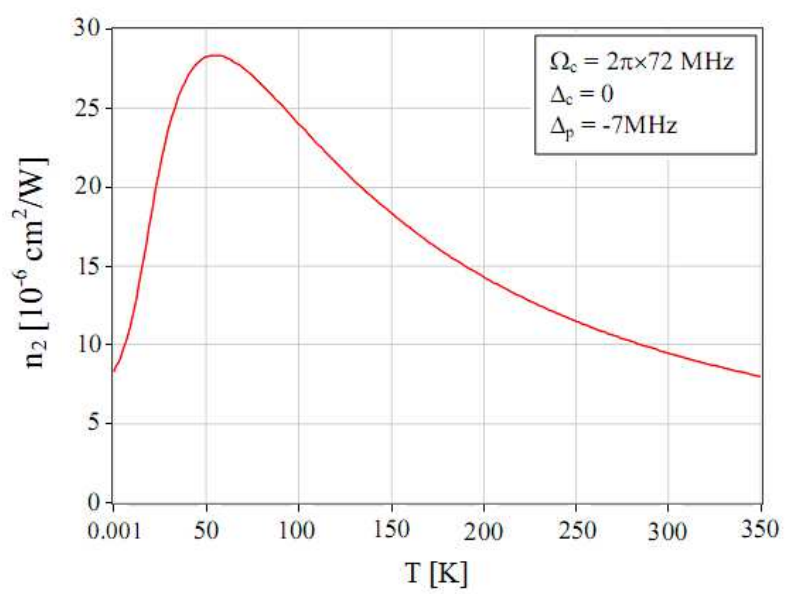

Fig. 5. Variation of the Kerr nonlinear coefficient versus absolute temperature in the case of $\Delta_{p}=-7 \mathrm{MHz}, \Delta_{p}=0$, and $\Omega_{c}=2 \pi \times 72 \mathrm{MHz}$. 


\section{CONCLUSION}

We have derived the analytical expression of the self-Kerr nonlinear coefficient $n_{2}$ of the three-level lambda system under Doppler broadening as function of controlling parameters of the coupling light, which is in good agreement with previous experimental observation. The analytical result obtained in this work not only gives sufficient knowledge on the Kerr nonlinearity but is also convenient to perform future studies relating such Kerr medium. It is shown that the Kerr nonlinearity of the three-level lambda EIT medium is greatly enhanced and depended on temperature of the medium. Furthermore, the magnitude, slop, and sign of the Kerr coefficient can be controlled by tuning frequency and/or intensity of the coupling light, or by changing tempera-

ture of the medium. Such greatly enhanced and controllable self-Kerr nonlinearity can be used to construct photonic devices working with low-light intensity at various temperature conditions.

\section{ACKNOWLEDGMENT}

The financial supports from the Vietnam's Ministry of Science and Technology (code 07/2012/HD-NDT) and the Vietnam's Ministry of Education and Training (code 08/2012/HDHTSP) are acknowledged.

\section{REFERENCES}

[1] K.J. Boller, A. Imamoglu, S.E. Harris, Phys. Rev. Lett. 66 (1991) 2593.

[2] S. E. Harris, J. E. Field, and A. Imamoglu, Phys. Rev. Lett. 64 (1990) 1107.

[3] S. E. Harris and L. V. Hau, Phys. Rev. Lett. 82 (1999) 4611.

[4] H. Schmidt and A. Imamogdlu, Opt. Lett. 21 (1996) 1936.

[5] V. Tikhonenko, J. Christou, and B. Luther-Davies, Phys. Rev. Lett., 76 (1996) 2698.

[6] Y. Li, and M. Xiao, Opt. Lett. 21 (1996) 1064.

[7] M. D. Lukin, S.F. Yelin, and M. Fleischhauer, Phys. Rev. Lett. 84 (2000) 4232.

[8] H. Wang, D. Goorskey, and M. Xiao, Phys.Rev.Lett. 87 (2001) 073601.

[9] A. Joshi and Min Xiao, Progress in Optics, Ed. E. Wolf, 49 (2006) 97-175.

[10] Daniel Adam Steck, $\mathrm{Rb}^{87}$ D Line Data: http://steck.us/alkalidata. 